\title{
WestVirginiaUniversity
}

THE RESEARCH REPOSITORY @ WVU

Graduate Theses, Dissertations, and Problem Reports

2003

\section{Generalized nowhere zero flow}

Jingjing Chen

West Virginia University

Follow this and additional works at: https://researchrepository.wvu.edu/etd

\section{Recommended Citation}

Chen, Jingjing, "Generalized nowhere zero flow" (2003). Graduate Theses, Dissertations, and Problem Reports. 1367.

https://researchrepository.wvu.edu/etd/1367

This Thesis is protected by copyright and/or related rights. It has been brought to you by the The Research Repository @ WVU with permission from the rights-holder(s). You are free to use this Thesis in any way that is permitted by the copyright and related rights legislation that applies to your use. For other uses you must obtain permission from the rights-holder(s) directly, unless additional rights are indicated by a Creative Commons license in the record and/ or on the work itself. This Thesis has been accepted for inclusion in WVU Graduate Theses, Dissertations, and Problem Reports collection by an authorized administrator of The Research Repository @ WVU. For more information, please contact researchrepository@mail.wvu.edu. 


\title{
Generalized Nowhere Zero Flow
}

\author{
Jingjing Chen \\ Thesis submitted to the \\ College of Engineering and Mineral Resources \\ at West Virginia University \\ in partial fulfillment of the requirements \\ for the degree of \\ Master of Science \\ in \\ Computer Science
}

Elaine M. Eschen, Ph.D., Chair

Frances L. Van Scoy, Ph.D.

Hong-Jian Lai, Ph.D.

Department of Computer Science

Morgantown, West Virginia

2003

Keywords: nowhere zero flow(NZF), group connectivity number(GCN), bipartite graph, chordal graph

Copyright 2003 Jingjing Chen 


\title{
ABSTRACT
}

\section{Generalized Nowhere Zero Flow}

\author{
Jingjing Chen
}

Let $G$ be an undirected graph, $A$ be an (additive) abelian group and $A^{*}=A-\{0\}$. A graph $G$ is $A$-connected if $G$ has an orientation $D(G)$ such that for every function $b: V(G) \mapsto A$ satisfying $\sum_{v \in V(G)} b(v)=0$, there is a function $f: E(G) \mapsto A^{*}$ such that at each vertex $v \in V(G), \partial f(v)$, the net flow out from $v$, equals $b(v)$. An $A$-nowhere-zero-flow (abbreviated as $A$ $\mathrm{NZF}$ ) in $G$ is a function $f: E(G) \mapsto A^{*}$ such that at each vertex $v \in V(G)$, $\partial f(v)=0$.

In this paper, we investigate the group connectivity number $\Lambda_{g}(G)=$ $\min \{n$ : if $A$ is an abelian group with $|A| \geq n$, then $G$ is $A$-connected $\}$ for certain families of graphs including complete bipartite graphs, chordal graphs, wheels and biwheels. We also give some general results and methods to approach nowhere zero flow and group connectivity problems. 


\section{Contents}

1 Introduction $\quad \mathbf{1}$

1.1 Definitions and Notations . . . . . . . . . . . . 1

1.2 An Example . . . . . . . . . . . . . . . . . . . . . 3

2 Preliminaries $\quad 6$

2.1 Chordless Cycles . . . . . . . . . . . . . . . . . 7

2.2 Collapsible Graphs . . . . . . . . . . . . . . . . . . . 7

2.3 Wheels . . . . . . . . . . . . . . . . 8

2.4 Complete Graphs . . . . . . . . . . . . . . . . . . . . . 8

2.5 Triangularly Connected Graphs . . . . . . . . . . . . 9

3 Methodologies $\quad \mathbf{1 0}$

3.1 Reductions of Graphs . . . . . . . . . . . . . . . . . 10

3.1.1 Vertex Splitting . . . . . . . . . . . . 10

3.1.2 Subgraph Contraction . . . . . . . . . . . . 11

3.2 Graph Attachment . . . . . . . . . . . . . . 13

4 Complete Bipartite Graphs 14

4.1 The Group Connectivity Number of $K_{m, 2}$. . . . . . . . . . . . 15

4.2 The Group Connectivity Number of $K_{m, 3} \ldots \ldots$. . . . . . . . . 17

4.3 The Group Connectivity Number of $K_{m, n},(m \geq n \geq 4)$. . . 18

5 Chordal Graphs $\quad 21$

6 The Biwheels $B_{n} \quad 25$

$\begin{array}{lll}7 & \text { General Graphs } & 27\end{array}$

$\begin{array}{llr}8 & \text { Algorithms } & 29\end{array}$

9 Conclusion $\quad 32$ 


\section{Chapter 1}

\section{Introduction}

\subsection{Definitions and Notations}

We start with some basic definitions. Let $G=(V, E)$ be an undirected graph with vertex set $V$ and edge set $E$, and $D$ be an orientation of the edge set $E$. Under the orientation $D$, an oriented edge is called an arc or a directed edge. When an $\operatorname{arc} e$ is oriented from a vertex $u$ to a vertex $v$, we call $u$ the tail of $e$ and $v$ the head of $e$. The graph $G$ under the orientation $D$ is sometimes denoted by $G^{\prime}$. Fix an orientation $D=D(G)$. For a vertex $v \in V$, let $E_{D}^{+}(v)$ (or $E_{D}^{-}(v)$, respectively) be the set all arcs of $G^{\prime}$ with their tails (heads, respectively) at vertex $v$. Let $A$ be a nontrivial abelian additive group. We shall use 0 or $0_{\mathrm{A}}$ to denote the identity element in $A$. Let $A^{*}=A-\{0\}$ and define

$$
F\left(G^{\prime}, A\right)=\left\{f: E\left(G^{\prime}\right) \mapsto A\right\} \text { and } F^{*}\left(G^{\prime}, A\right)=\left\{f: E\left(G^{\prime}\right) \mapsto A^{*}\right\} .
$$

For each $f \in F\left(G^{\prime}, A\right)$, the boundary of $f$ is a function $\partial f: V(G) \mapsto A$ defined by

$$
\partial f(v)=\sum_{e \in E^{+}(v)} f(e)-\sum_{e \in E^{-}(v)} f(e),
$$

where " $\sum$ " refers to the addition in $A$. Throughout this paper, we shall adopt the following convenience: if $X \subseteq E(G)$ and $f: X \mapsto A$ is a function, then we regard $f$ as a function $f: E(G) \mapsto A$ such that $f(e)=0$ for all $e \in E(G)-X$.

An $A$-nowhere-zero-flow (abbreviated as $A$-NZF) in $G^{\prime}$ is a function $f \in F^{*}\left(G^{\prime}, A\right)$ such that $\partial f=0$. The nowhere-zero-flow problems were introduced by Tutte [20], and recently surveyed by Jaeger in [7].

A function $b: V(G) \mapsto A$ is called an A-valued zero sum function on $G$ if $\sum_{v \in V(G)} b(v)=0$ in $G$. The set of all A-valued zero sum functions on 
$G$ is denoted by $Z(G, A)$. Given $b \in Z(G, A)$ and orientation $G^{\prime}$, a function $f \in F^{*}\left(G^{\prime}, A\right)$ is an $(A, b)$-nowhere zero flow $((A, b)$-NZF $)$ if $\partial f=b$. A graph $G$ is $A$-connected if $G$ has an orientation $G^{\prime}$ such that for every $b, G$ has an $(A, b)$-NZF. For an abelian group $A$, let $\langle A\rangle$ be the family of graphs that are $A$-connected. It is observed in [8] that the property $G \in\langle A\rangle$ is independent of the orientation of $G$. Since if it is satisfied for one orientation of $G$, then it holds for every orientation (replace $f(e)$ by $-f(e)$ if the orientation of an edge $e$ is reversed).

The concept of $A$-connectivity was introduced by Jaeger et al. in [8], where $A$-NZFs were successfully generalized to $A$-connectivities. A concept similar to the group connectivity was independently introduced in [9], with a different motivation from [8].

From the definition, we see the $A$-connectivity is a more generalized concept than $A$-NZF. A big difference is their monotonicity. A fundamental theorem in the theory of NZFs states that the existence of an $A-\mathrm{NZF}$ in a graph $G$ depends only on the order of the abelian group $A[20]$. Thus, " $G$ admits a $k$-NZF" is used to indicate that for every abelian group of order $k$ there exists an $A$-NZF in $G$. The monotonicity property of NZFs stated in [20] is: If $G$ admits a $k$-NZF then it admits a $t$-NZF for every $t>k$. But a similar statement with respect to $A$-connectivity is false. In fact, Jaeger et al. [8] presented an example showing that a graph $G$ can be $Z_{5}$-connected but not $Z_{6}$-connected.

The motivation of this paper is as follows: For a given graph $G$, we want to determine the smallest integer $k$ such that for each abelian group $A$ with $|A| \geq k, G$ is $A$-connected.

The number $\Lambda_{g}(G)=\min \{k:$ if $A$ is an abelian group with $|A| \geq k$, then $G \in\langle A\rangle\}$ will be called group connectivity number, or GCN for short, of a certain graph $G$.

A weaker problem has been considered by Shahmohamad [19]: Given a graph $G$, determine the smallest integer $\lambda$ for which $G$ has a nowhere zero $\lambda$-flow. A $\lambda$-flow of a graph $G$ is a flow $f$ such that $|f(e)|<\lambda$ for every edge edge $e \in E(G)$ and for every vertex $v \in V(G)$

$$
\sum_{e \in E^{+}(v)} f(e) \equiv \sum_{e \in E^{-}(v)} f(e) \quad(\bmod \lambda) .
$$

Let $\lambda_{g}(G)$, the minimum flow number, denote this minimum $\lambda$. The minimum flow number of NZF of a graph is based on the stronger condition of local balance at every vertex (that is, when $b_{\mathbf{A}}=0$ ). We generalize the results stated in [19] by extending some of the results in [19] on $\lambda_{g}(G)$ to 
their group connectivity counterpart on $\Lambda_{g}(G)$.

In this paper, we determine best possible upper bounds for the GCN on the following families of graphs: complete bipartite graphs, chordal graphs, wheels and biwheels, and characterize the extremal graphs.

This paper is organized as follows: In Chapter 2 former related results and properties on group connectivity are presented. Some methodologies about graph reduction will be developed in Chapter 3. All these properties and methods will be used to prove the main results. We prove the main results in Chapter 4 through Chapter 6 . In Chapter 7 we give the famous conjectures and results in this area on general graphs. And we will give algorithms to decide the A-connectivity of chordal graphs, complete bipartite graphs and general graphs in Chapter 8. We give conclusions in the last chapter.

\subsection{An Example}

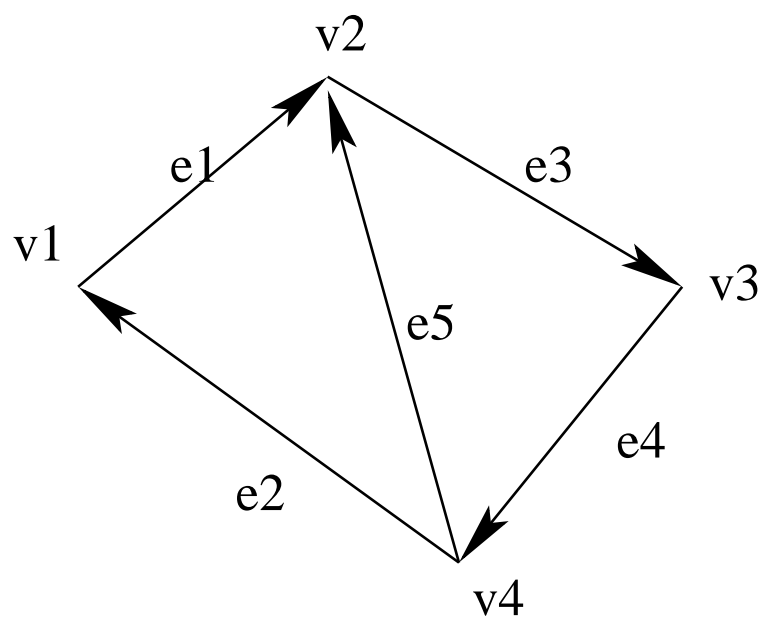

Figure 1.1: A directed graph

Figure 1.1 shows a digraph with $V(G)=\left\{v_{1}, v_{2}, v_{3}, v_{4}\right\}$ and $E(G)=$ $\left\{e_{1}, e_{2}, e_{3}, e_{4}, e_{5}\right\}$ with $e_{1}=\left(v_{1}, v_{2}\right), e_{2}=\left(v_{4}, v_{1}\right), e_{3}=\left(v_{2}, v_{3}\right), e_{4}=\left(v_{3}, v_{4}\right)$, $e_{5}=\left(v_{4}, v_{2}\right)$.

This way (listing out all vertices and existing edges) of describing a graph is quite cumbersome. A more succinct description employs matrices. We construct a matrix whose rows are labelled by the vertices and whose columns are labelled by the edges. If there is an edge $e_{k}$ oriented from $v_{i}$ to $v_{j}$, we set the $(i, k)$-entry of the matrix to +1 and $(j, k)$ to -1 . The rest of the entries in the $k$ th column of the matrix are zero. 
For example, the graph shown in Figure 1.1 can be represented by the following $4 \times 5$ incidence matrix

$$
M_{D}=\begin{aligned}
& e_{1} \\
& v_{1} \\
& v_{2} \\
& v_{3} \\
& v_{4}
\end{aligned}\left(\begin{array}{ccccc}
1 & -1 & 0 & 0 & 0 \\
-1 & 0 & 1 & 0 & -1 \\
0 & 0 & -1 & 1 & 0 \\
0 & 1 & 0 & -1 & 1
\end{array}\right)
$$

Notice that in general this is not necessarily a square matrix. In each row, the nonzero entries represents the edges incident on the corresponding vertex, and each column contains exactly two nonzero entries, one being +1 (representing the tail of the edge) and the other -1 (representing the head of the edge).

The next step is to flush some 'liquid' into the graph, so that there is a flow in the graph. Mathematically, this is equivalent to associating each edge with a non-zero weight $f(e)$. The weight could be an element in an abelian group (such as a real number, or an integer). Thus, a flow can be viewed as a mapping $f$ with domain $E(G)$. In this paper, we are specially interested in the particular case when $f$ takes value in an abelian group $A$. For a positive integer $n, Z_{n}$ denotes the cyclic group on $n$ elements. For convenience we write $Z_{3}=\{0,1,2\}$, and $Z_{4}=\{0,1,2,3\}$. So for example, a flow in $Z_{3}$ is actually even simpler than you might have expected: there are just three flow values that are denoted by elements in $\{0,1,2\}$. Finally, we call a flow nowhere zero if zero is excluded from the value taken by $f$.

In the matrix language, a flow is represented by a column vector $f_{\mathbf{A}}$, where the subscript ' $A$ ' emphasizes the abelian group. A flow for the graph in Figure 1.1 can be written as

$$
\left.\begin{array}{rl|l}
f_{\mathbf{A}}= & e_{1} & f\left(e_{1}\right) \\
e_{2} & e_{3} & f\left(e_{2}\right) \\
& e_{4} & f\left(e_{3}\right) \\
& e_{5} & f\left(e_{4}\right)
\end{array}\right) .
$$

$f_{\mathbf{A}}$ is an $A$-flow of $G$ if $M_{D} \cdot f_{\mathbf{A}}=0$. A nowhere zero flow will be denoted with an asterisk ' $*$ ', $f_{\mathbf{A}}^{*}$.

From the view point at a vertex $v_{i}$, the oriented edges incident with it could be either incoming or outgoing. The net out-flow $b_{i}$ of a vertex $v_{i}$ is an indication of local flow balance around $v_{i}$. For example, the net out-flow of vertex $v_{2}$ in Figure 1.1 can be calculated as

$$
b_{\mathbf{A}}\left(v_{2}\right)=f_{\mathbf{A}}\left(e_{3}\right)-f_{\mathbf{A}}\left(e_{1}\right)-f_{\mathbf{A}}\left(e_{5}\right) .
$$


If this number is zero (in the designated abelian group), we say local balance is achieved at $v_{2}$. If local balance is achieved at all vertices in $G$, then $f$ is an $A$-nowhere-zero-flow $(A$-NZF). A less restricted condition, global balance, refers to the case where the sum of net out-flows over all vertices vanishes (in the abelian group), that is

$$
\sum_{v_{i} \in V(G)} b_{\mathbf{A}}\left(v_{i}\right)=0_{\mathbf{A}}
$$

One of the advantages of using the matrix language is to compute net out-flows for all vertices at once: It is simply the product of the incidence matrix and the flow vector:

$$
M_{D} \cdot f_{\mathbf{A}}=b_{\mathbf{A}}
$$

Notice that the dimension of $b$ is the number of vertices while the dimension of $f$ is the number of edges. If local balance is achieved at all vertices, then (1.5) is a system of homogeneous equations. In general (1.5) is a system of non-homogeneous equations.

Stated in a simpler language, the question is that given a matrix $M_{D}$, does every vector $b$ with $\sum b_{\mathbf{A}}=0_{\mathbf{A}}$ have a solution for $f_{\mathbf{A}}^{*}$ in (1.5)? If the answer to the above question is 'yes', we say that the graph $G$ is $A$-connected, and write $G \in\langle A\rangle$, where $\langle A\rangle$ denotes the family of graphs that are $A$-connected.

Shahmohamad [19] did not use the matrix language. Recaptured in the matrix language, the results [19] of the minimum flow number $\lambda_{g}(G)$ are for the case of homogeneous systems of equations. Here, we consider the non-homogeneous cases and generalize the results to the group connectivity number $\Lambda_{g}(G)$. 


\section{Chapter 2}

\section{Preliminaries}

Before we embark on the main problem, we want to consider some simple cases. The aim is two-folded: first, we can build up our knowledge as well as our confidence through working on the simpler cases. Secondly, and more importantly, the conclusions of these cases themselves can be used as grounding place to tackle on more complex problems.

First we introduce the concept of connectivity of a graph. A vertex cut of a graph $G$ is a subset $V^{\prime}$ of $V$, such that $G-V^{\prime}$ is disconnected or $G-V^{\prime}=K_{1}$. A $k$-vertex cut is the vertex cut of $k$ elements. The connectivity $\kappa(G)$ of $G$ is the minimum positive integer $k$ for which $G$ has a $k$-vertex cut; $G$ is said to be $k$-connected if $\kappa(G) \geq k$. Similarly, an edge cut of $G$ is a subset of $E$ of the form $[S, \bar{S}]$, where $S$ is a non-empty proper subset of $V, \bar{S}=V-S$ and where $[S, \bar{S}]$ denotes the set of edges in $G$ with one end in $S$ and the other end in $\bar{S}$. A $k$-edge cut is an edge cut of $k$ elements. If $G$ is nontrivial (a graph $G$ is nontrivial if $G$ is connected with $|V(G)| \geq 2$ ) and $E^{\prime}$ is an edge cut of $G$, then $G-E^{\prime}$ is disconnected. We define the edge connectivity $\kappa^{\prime}(G)$ of $G$ to be the minimum positive integer $k$ for which $G$ has a $k$-edge cut. It is a well-known conclusion in graph theory that $\kappa(G) \leq \kappa^{\prime}(G) \leq \delta(G)$, where $\delta(G)$ stands for the minimum degree of $G$.

Proposition 2.1 (Zhang [22]) Let $G \in\langle A\rangle$ for any $A$. Then $\kappa^{\prime}(G) \geq 2$.

The condition of bridgelessness (no cut edge) is necessary for graph admitting nowhere zero flows.

Proposition 2.2 (Jaeger et al. [8]) Let $G$ be a connected graph with $n$ vertices and $m$ edges. Then $\Lambda_{g}(G)=2$ if and only if $n=1$ (and so $G$ has $m$ loops with a single vertex). 
No graph except $K_{1}$ is $Z_{2}$-connected. There is only one function in $F^{*}\left(G, Z_{2}\right)$ and its boundary, the degree parity function on $V$, is not the only zero sum function on $G$, unless $|V|=1$.

We need the notion of graph contraction. Let $G$ be a graph and let $X \subseteq E(G)$ be an edge subset. The contraction $G / X$ is the graph obtained from $G$ by identifying the two ends of each edge $e$ in $X$ and deleting $e$. If $X=\{e\}$, then we write $G / e$ for $G /\{e\}$. If $H$ is a subgraph of $G$, then we write $G / H$ for $G / E(H)$. Note that even if $G$ is a simple graph, the contraction $G / X$ may have loops and multiple edges. We have the following properties of graph contraction:

Proposition 2.3 (Lai [11]) Let $A$ be an abelian group. Then $\langle A\rangle$ satisfies each of the following:

(C1) $K_{1} \in\langle A\rangle$.

(C2) If $G \in\langle A\rangle$ and $e \in E(G)$, then $G / e \in\langle A\rangle$.

(C3) If $H$ is a subgraph of $G$ and if both $H \in\langle A\rangle$ and $G / H \in\langle A\rangle$, then $G \in\langle A\rangle$.

\subsection{Chordless Cycles}

An edge $e$ is called a chord of a cycle if $e$ is not an edge of the cycle and both end vertices of $e$ are in the cycle. A chordless cycle $C_{n}$ is a cycle without chord. For this important class of graphs, there is a simple result:

Lemma 2.4 (Lai [11]) Let $C_{n}$ denote a cycle of $n(\geq 1)$ vertices and let $A$ be an abelian group. Then $C_{n} \in\langle A\rangle$ if and only if $|A| \geq n+1$.

\subsection{Collapsible Graphs}

Let $O(G)=$ odd degree vertices of $G\}$. A graph $G$ is collapsible if for any subset $R \subseteq V(G)$ with $|R| \equiv 0(\bmod 2), G$ has a spanning connected subgraph $\Gamma_{R}$ such that $O\left(\Gamma_{R}\right)=R$. A tree is a connected acyclic graph. A spanning tree is a spanning subgraph of $G$ that is a tree.

Theorem 2.5 (Catlin [2]) Suppose that $G$ is one edge short of having two edge-disjoint spanning trees. Then $G$ is collapsible if and only if $\kappa^{\prime}(G) \geq 2$.

Lemma 2.6 (Lai [10]) Let $G$ be a collapsible graph and let $A$ be an abelian group with $|A|=4$. Then $G \in\langle A\rangle$. 


\subsection{Wheels}

A wheel $W_{m}$ is a graph obtained from a cycle with $m$ edges by adding a new vertex $v$ and then joining this new vertex to all the vertices on the cycle. This new vertex $v$ is called the center of $W_{m}$. For the wheels, we have the following result:

Lemma 2.7 (Lai [11]) Let $W_{m}$ be the wheel of $m+1$ vertices. Then $W_{4} \in$ $\langle A\rangle$, for any Abelian group $A$ with $|A| \geq 3$.

Lemma 2.8 (Lai, Xu and Zhang [14]) (1) $W_{2 n} \in\left\langle Z_{3}\right\rangle$.

(2) Let $G \cong W_{2 n+1}, b \in Z\left(G, Z_{3}\right)$. Then there exists a $\left(Z_{3}, b\right)-N Z F$ $f \in F^{*}\left(G, Z_{3}\right)$ if and only if $b \neq 0$.

If $|A| \geq 4$, we can contract $C_{2}$ and $C_{3}$ from $W_{2 n}$. It follows by Lemma 2.4 and Proposition 2.3 that $W_{2 n} \in\langle A\rangle$. Furthermore by above Lemma 2.8, we know that $W_{2 n} \in\left\langle Z_{3}\right\rangle$. Thus we have the following Lemma:

Lemma 2.9 $W_{2 n} \in\langle A\rangle$, for any Abelian group $A$ with $|A| \geq 3$.

\subsection{Complete Graphs}

A complete graph is a simple graph in which each pair of distinct vertices is joined by an edge. The complete graph on $n$ vertices is denoted by $K_{n}$.

Lemma 2.10 (Tutte [21]) Let $G$ be a 3-regular graph, then $G$ has a $Z_{3}-N Z F$ if and only if $G$ is bipartite.

The complete graph $K_{4}$ is a 3-regular graph (each vertex has degree 3 ). But it is obvious not a bipartite graph. Since $K_{4}$ has 2 edge-disjoint spanning trees, follows from Theorem 3.1 of Jaeger et al. [8] which stated that every graph which contains 2-edge-disjoint spanning trees is $A$-connected for every abelian group $A$ with order $|A| \geq 4$, we have the following:

Lemma 2.11 $K_{4} \in\langle A\rangle$, for any Abelian group with $|A| \geq 4$.

Shahmohamad ([18] and [19]) gave the following results on minimum flow number of complete graphs:

Lemma 2.12 ([18], [19]) Let $n$ be a positive integer.

(1) $\lambda_{g}\left(K_{2 n+1}\right)=2$, for $n \geq 1$.

(2) $\lambda_{g}\left(K_{2 n}\right)=3$, for $n \geq 3$. 
We generalized the above results to the group connectivity number of complete graphs as follows:

Lemma 2.13 Let $n \geq 3$ be an integer. Then

$$
\Lambda_{g}\left(K_{n}\right)= \begin{cases}4 & \text { if } 3 \leq n \leq 4 \\ 3 & \text { if } n \geq 5\end{cases}
$$

Proof. $\quad$ By Lemma 2.4, $\Lambda_{g}\left(K_{3}\right)=4$. By Proposition 2.2, $\Lambda_{g}\left(K_{4}\right) \neq 2$. By Lemma $2.9, K_{4}$ does not have a $Z_{3}-\mathrm{NZF}$, and therefore cannot be $Z_{3^{-}}$ connected. Thus, $\Lambda_{g}\left(K_{4}\right) \neq 3$. Then by Lemma 2.11, $\Lambda_{g}\left(K_{4}\right)=4$. When $n \geq 5$, the result follows from Proposition 2.3(C3), Lemma 2.7 and Lemma 2.2 since $K_{n}$ contains a $W_{n-1}$ which when contracted yields a single vertex with self-loops.

\subsection{Triangularly Connected Graphs}

A graph $G$ is triangularly connected [14] if for every e, $f \in E(G)$, there exists a sequence of cycles $c_{1}, c_{2}, \ldots, c_{k}$ such that $e \in E\left(c_{1}\right), f \in E\left(c_{k}\right),\left|E\left(c_{i}\right)\right| \leq 3$ for $1 \leq i \leq k$, and $E\left(c_{j}\right) \cap E\left(c_{j+1}\right) \neq \emptyset$ for $1 \leq j \leq k-1$.

Lemma 2.14 Let $G$ be a triangularly connected graph, if $H$ is a nontrivial subgraph of $G$ and $H \in\left\langle Z_{3}\right\rangle$, then $G \in\left\langle Z_{3}\right\rangle$.

Proof. First assume that $H$ is not a spanning subgraph of $G$. Since $H$ is nontrivial and since $G$ is triangularly connected, $G / H$ must contain a $C_{2}$. Since $G$ is a triangularly connected graph, we keep contracting $C_{2}$ until we finally obtain one vertex. By Lemma $2.4 C_{2}$ is in $\left\langle Z_{3}\right\rangle$, and so by Proposition 2.3, we must have $G \in\left\langle Z_{3}\right\rangle$. Otherwise $G / H$ is a single vertex graph and so it is in $\left\langle Z_{3}\right\rangle$ by Proposition 2.2, and then by Proposition 2.3, $G \in\left\langle Z_{3}\right\rangle$. 


\section{Chapter 3}

\section{Methodologies}

We have gotten some good tastes of the problem in some simple cases in the previous chapter. Those examples have the virtue of being simple, and thus solvable via a 'brute force' method. The majority of the remaining cases we consider, however, are not as simple, and a direct brute force method often proves to be difficult, if not impossible. In this chapter, we develop some systematic methods, which can be used efficiently in dealing with more general graphs.

\subsection{Reductions of Graphs}

The simple, and often extremely useful strategy is to reduce a large, complicated graph into smaller and simpler pieces, and to deal with each piece one at a time. However, caution must be taken when we carry out the reduction, for some operations may be illegal as the GCN of the reduced graph may not be the same as that of the original graph.

In the following, we discuss two types of reduction operations, each of which, when carried out with care, leave $\Lambda_{g}$ unchanged.

\subsubsection{Vertex Splitting}

The 'Vertex Splitting' or SP reduction refers to the process of splitting one vertex $v$, while eliminating two edges $e_{1}$ and $e_{2}$ (we do not consider the direction of the edges at this time), each of which is incident on $v$, and creating a new edge between $v_{1}$ and $v_{2}$ (see Figure3.1).

The SP reduction is not very messy, as you can see from the resulting graph, denoted by $G_{\Delta}\left\{e_{1}, e_{2}\right\}$, which still has the same number of vertices, 


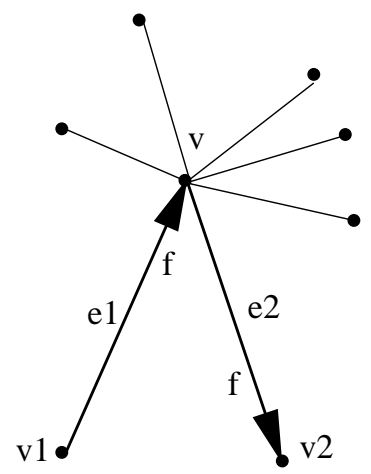

(a)

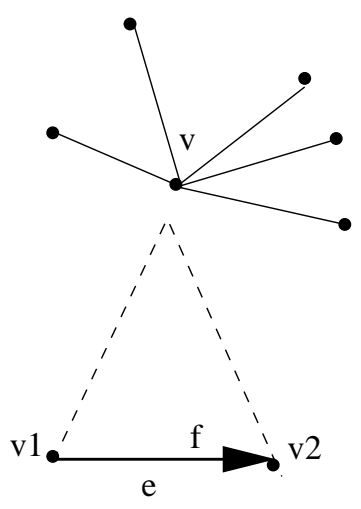

(b)

Figure 3.1: Vertex Splitting

and only one edge fewer than $G$ has. What is useful about this reduction, however, is that it sometimes changes the structure of the original graph considerably. This is especially true when the original graph has many long cycles: applying SP in this case could effectively reduce the size of the cycle. Also note that the SP reduction of a simple graph (without double edges) may not necessarily be simple any more.

Theorem 3.1 $\Lambda_{g}(G) \leq \Lambda_{g}\left(G_{\Delta}\left\{e_{1}, e_{2}\right\}\right)$.

The above theorem tells that we will always find an upper bound of $\Lambda_{g}(G)$ in the reduced graph. If we are lucky, we may find the true value of $\Lambda_{g}(G)$.

The proof is relatively straightforward. For any $b \in Z(G, A)$, since $V(G)=V\left(G_{\Delta}\left\{e_{1}, e_{2}\right\}\right)$, we can view $b \in Z\left(G_{\Delta}\left\{e_{1}, e_{2}\right\}, A\right)$ as well. Suppose there is an $(A, b)$-NZF $f \in F^{*}\left(G_{\Delta}\left\{e_{1}, e_{2}\right\}, A\right)$ such that $\partial f=b$. Then we can assign the same value $f(e)$ to the edge $e_{1}$ and $e_{2}$ (see Figure 3.1(a)), the value of $b(v), b\left(v_{1}\right), b\left(v_{2}\right)$ will not be changed. Thus, when $G_{\Delta}\left\{e_{1}, e_{2}\right\}$ (see Figure $3.1(\mathrm{~b})$ ) is $A$-connected, so is $G$.

\subsubsection{Subgraph Contraction}

Another type of reduction, which we call subgraph contraction or simply contraction, uses a single vertex to represent a subset of vertices in the original graph. More specifically, a contraction does the following: Let $H$ be a subgraph of the graph $G$. Eliminate all edges within $H$. Then 'squeeze' all vertices in $H$ into one vertex $v_{H}$. Any edge originally connecting $u_{1} \notin H$ and

$v_{1} \in H$ now becomes $e^{\prime}\left[u_{1}, v_{H}\right]$ (see Figure 3.2). The reduced graph is noted 
by $G / H$. Note that even when $G$ is simple, $G / H$ may have multiple edges or loops.

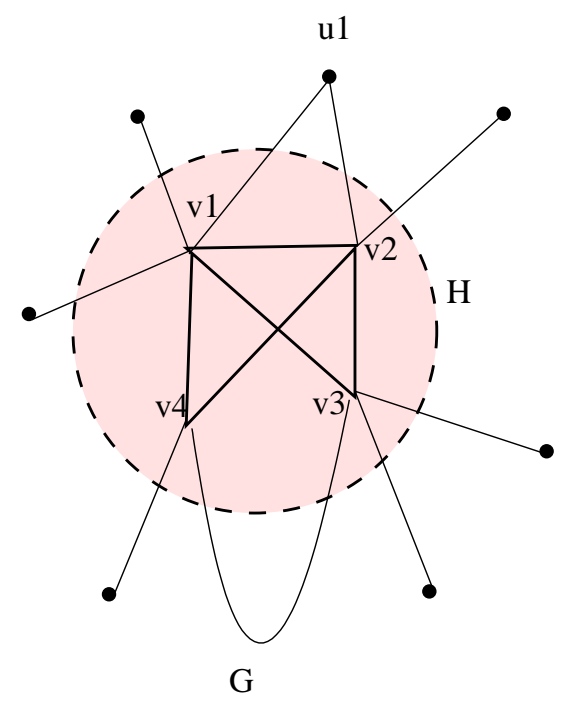

Edge [v4, v3] is not in $\mathrm{H}$

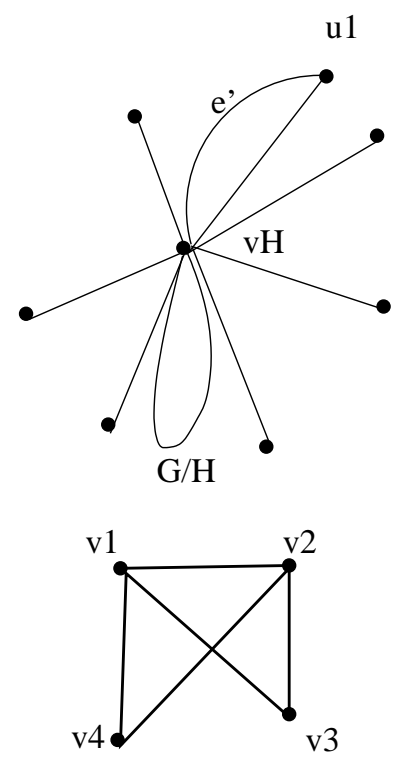

$\mathrm{H}$

Figure 3.2: Subgraph Contraction

Theorem 3.2 Let $H$ be a 2-edge-connected subgraph of $G$. If $G / H$ has more than one vertex, then $\Lambda_{g}(G) \geq \Lambda_{g}(G / H)$.

Proof. Fix an abelian group $A$ with $|A| \geq \Lambda_{g}(G)$. Let $b^{\prime} \in Z(G / H, A)$, and $v_{H}$ be the vertex in $G / H$ onto which $H$ is contracted. Fix a vertex $v_{0} \in V(H)$. Define $b \in Z(G, A)$ as follows

$$
b(z)=\left\{\begin{array}{ll}
b^{\prime}(z) & \text { if } z \in V(G)-V(H) \\
b^{\prime}\left(v_{H}\right) & \text { if } z \in v_{0} \\
0 & \text { if } z \in V(H)-\left\{v_{0}\right\}
\end{array} .\right.
$$

Then

$$
\sum_{z \in V(G)} b(z)=\sum_{z \in V(G / H)} b(z)=0
$$

and so $b \in Z(G, A)$ indeed.

Since $|A| \geq \Lambda_{g}(G)$, there is a function $f \in F^{*}(G, A)$ such that $\partial f=b$. Let $A_{G}(H)=\{z \in V(H): z$ is incident with an edge in $E(G)-E(H)\}$ and let $f^{\prime}$ be the restriction of $f$ on $E(G)-E(H)=E(G / H)$. Then at $v_{H}$, 


$$
\begin{aligned}
\partial f^{\prime}\left(v_{H}\right) & =\sum_{e \in E_{G / H}^{+}\left(v_{H}\right)} f^{\prime}(e)-\sum_{e \in E_{G / H}^{-}\left(v_{H}\right)} f^{\prime}(e) \\
& =\sum_{v \in A_{G}(H)}\left(\sum_{e \in E_{G}^{+}(v)} f(e)-\sum_{e \in E_{G}^{-}(v)} f(e)\right) \\
& =\sum_{v \in A_{G}(H)} \partial f(v) .
\end{aligned}
$$

Since $\partial f=b, b\left(v_{0}\right)=b^{\prime}\left(v_{H}\right) \quad$ and $\quad \forall z \in V(H)-\left\{v_{0}\right\}, b(z)=0$,

$$
\partial f^{\prime}\left(v_{H}\right)=\sum_{v \in A_{G}(H)} \partial f(v)=\sum_{v \in V(H)} \partial f(v)=\partial f\left(v_{0}\right)=b^{\prime}\left(v_{H}\right) .
$$

On the other hand, for any $z \in V(G / H)-\left\{v_{H}\right\}, \partial f^{\prime}(z)=\partial f(z)=$ $b(z)=b^{\prime}(z)$. Hence $\partial f^{\prime}=b^{\prime}$ and so $f^{\prime}$ is an $\left(A, b^{\prime}\right)-\mathrm{NZF}$ of $G / H$. This implies $\Lambda_{g}(G) \geq \Lambda_{g}(G / H)$.

Theorem 3.3 If $H$ is a 2-edge-connected subgraph of $G$, then $\Lambda_{g}(G) \leq$ $\max \left(\Lambda_{g}(H), \Lambda_{g}(G / H)\right)$.

Proof. If $\Lambda_{g}(H) \geq \Lambda_{g}(G)$, then $\Lambda_{g}(G) \leq \Lambda_{g}(H) \leq \max \left(\Lambda_{g}(H), \Lambda_{g}(G / H)\right)$, and so we may assume that $\Lambda_{g}(H)<\Lambda_{g}(G)$.

(1) If $\Lambda_{g}(H) \leq \Lambda_{g}(G / H)$, then let $A$ be an abelian group with $|A| \geq$ $\Lambda_{g}(G / H)$. Hence $H \in\langle A\rangle$ and $G / H \in\langle A\rangle$. By Proposition 2.3(C3), $G \in\langle A\rangle$ also. Therefore $\Lambda_{g}(G) \leq \Lambda_{g}(G / H)$. By Theorem 3.2, $\Lambda_{g}(G)=\Lambda_{g}(G / H)$ in this case.

(2) If $\Lambda_{g}(H)>\Lambda_{g}(G / H)$, then let $A$ be an abelian group with $|A| \geq$ $\Lambda_{g}(H)$. Hence $H \in\langle A\rangle$ and $G / H \in\langle A\rangle$. By Proposition 2.3(C3), $G \in\langle A\rangle$ also. Therefore $\Lambda_{g}(G) \leq \Lambda_{g}(H)$, which contradicts to the assumption that $\Lambda_{g}(H)<\Lambda_{g}(G)$, so no such subcase exists.

\subsection{Graph Attachment}

Let $G$ be a graph with $u^{\prime} v^{\prime} \in E(G)$ and $H$ be a graph with $u v \in E(H)$. We use $G \oplus H$ to denote a new graph obtained from the disjoint union of $G-\left\{u^{\prime} v^{\prime}\right\}$ and $H$ by identifying $u^{\prime}$ and $u$ and identifying $v^{\prime}$ and $v$. This operation is called attaching $G$ on $H$ over the edge $u v$ and the resulting graph is denoted by $G \oplus H$.

Lemma 3.4 (Devos et al. [4]) Let $G_{1}, G_{2}$ be graphs and let $H=G_{1} \oplus G_{2}$. If neither $G_{1}$ nor $G_{2}$ is $Z_{3}$-connected, then $H$ is not $Z_{3}$-connected. 


\section{Chapter 4}

\section{Complete Bipartite Graphs}

A complete bipartite graph consists of two sets of vertices: There is no edge joining two vertices within each set of vertices, but between each pair of interset vertices there is always one edge. This class of graphs are noted as $K_{m, n}$, where $m$ and $n$ are the numbers of the vertices in the two sets respectively.

Shahmohamad [19] gave the following results on minimum flow number of complete bipartite graphs:

Lemma 4.1 ([18], [19]) Let $m, n$ be positive integers.

(1) $\lambda_{g}\left(K_{m, n}\right)=2$, for $m$ and $n$ both even.

(2) $\lambda_{g}\left(K_{m, n}\right)=3$, for $m$ and $n$ are not both even integers.

We will generalize these results to the group connectivity number. In this chapter, we shall determine $\Lambda_{g}\left(K_{m, n}\right)$. By Proposition 2.1 we will only consider complete bipartite graphs with $m \geq n \geq 2$.

Proposition 4.2 Let $H$ be a graph on 2 vertices with $n$ ( $\geq 2$ ) edges joining these two vertices. Then $\Lambda_{g}(H)=3$.

Proof. Let $E(H)=\left\{e_{1}, e_{2}, \ldots, e_{n}\right\}$ with $n \geq 2$, and let $C_{2}=H\left[\left\{e_{1}, e_{2}\right\}\right]$ be a 2-cycle. By Lemma 2.4, $C_{2} \in\langle A\rangle$ with $|A| \geq 3$. Since $H / C_{2}$ is a single vertex with $n-2$ loops, by Proposition $2.2, \Lambda_{g}\left(H / C_{2}\right)=2<3$, and so $H / C_{2} \in\langle A\rangle$ with $|A| \geq 3$. By Proposition 2.3, $H \in\left\langle Z_{3}\right\rangle$.

It is trivial to see that if $m \geq n \geq 2$, a complete bipartite graph $K_{m, n}$ has a subgraph isomorphic to $K_{m-1, n}$, and $K_{m, n} / K_{m-1, n}$ is a graph with two vertices and $n \geq 2$ edges. By Proposition $4.2, \Lambda_{g}\left(K_{m, n} / K_{m-1, n}\right)=3$. Thus, by Theorem 3.3, we have an upper bound on $\Lambda_{g}\left(K_{m, n}\right)$ : 
Theorem 4.3 For any $m \geq n \geq 2, \Lambda_{g}\left(K_{m, n}\right) \leq \max \left(\Lambda_{g}\left(K_{m-1, n}\right), 3\right)$.

similarly we have $\Lambda_{g}\left(K_{m-1, n}\right) \leq \max \left(\Lambda_{g}\left(K_{m-2, n}\right), 3\right)$, repeat this induction until $m$ reaches $n$, we have the following Corollary:

Corollary of Theorem 4.3: $\Lambda_{g}\left(K_{m, n}\right) \leq \max \left(\Lambda_{g}\left(K_{n, n}\right), 3\right)$.

For the rest of the chapter, we will mostly concentrate on establishing a lower bound on $\Lambda_{g}\left(K_{m, n}\right)$, especially for cases of $K_{n, n}$.

\subsection{The Group Connectivity Number of $K_{m, 2}$}

Theorem $4.4 \Lambda_{g}\left(K_{m, 2}\right)=5$ for any integer $m \geq 2$.

Proof. Note that $K_{2,2}$ is isomorphic to the chordless cycle $C_{4}$. By Lemma 2.4, we have

$$
\Lambda_{g}\left(K_{2,2}\right)=5 .
$$

Thus, by Theorem 4.3,

$$
\Lambda_{g}\left(K_{m, 2}\right) \leq 5
$$

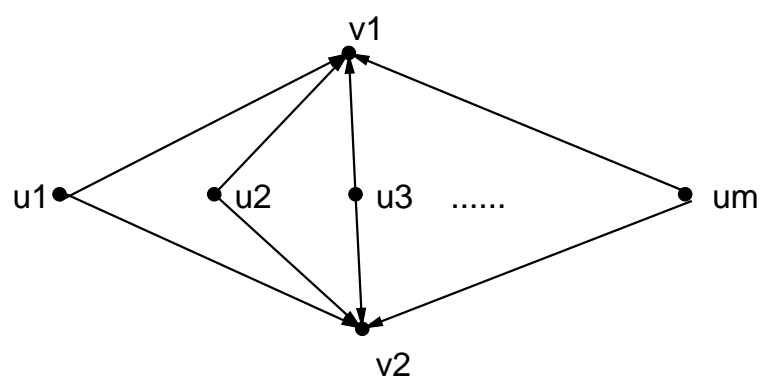

Figure 4.1: $K_{m, 2}$

Next, we want to show that

$$
\Lambda_{g}\left(K_{m, 2}\right)>4 .
$$

We will prove Inequality (4.3) by providing two counter examples. Let $A=$ $\left\{0, a_{1}, a_{2}, a_{3}\right\}$ be an abelian group, where $a_{2}$ is an element of order 2 . Assume (falsely) $K_{m, 2} \in\langle A\rangle$. Thus for each $b \in Z(A, G)$, one can always find $f \in$ $F^{*}(G, A)$ such that

$$
\partial f=b .
$$


Using the notation in Figure 4.1, we consider any function $b: V(G) \mapsto A$ such that $b\left(u_{1}\right)=b\left(u_{2}\right)=\ldots=b\left(u_{m}\right)=a_{2}$.

Orient each edge in this $K_{m, 2}$ from a $u_{i}$ to a $v_{j}$ and denote $f\left(u_{i}, v_{j}\right)=f_{i j}^{*}$. Thus,

$$
f_{i 1}^{*}+f_{i 2}^{*}=b\left(u_{i}\right)=a_{2} \text {, for each } i=1,2, \cdots, m .
$$

We know there are two abelian groups with 4 elements: $Z_{4}$ and $Z_{2} \times Z_{2}$. We will discuss them separately:

Case 1: Suppose that $A=Z_{4}$. Equation (4.5) above has solution either $f_{i 1}^{*}=f_{i 2}^{*}=a_{1}$ or $f_{i 1}^{*}=f_{i 2}^{*}=a_{3}$. It follows by (4.4) that:

$$
b\left(v_{1}\right)=-\sum_{i=1}^{m} f_{i 1}^{*}=-\sum_{i=1}^{m} f_{i 2}^{*}=b\left(v_{2}\right) .
$$

Now if we set $b\left(v_{1}\right)=a_{1} \neq b\left(v_{2}\right)=a_{3}$ when $m$ is even, and set $b\left(v_{1}\right)=0 \neq$ $b\left(v_{2}\right)=a_{2}$ when $m$ is odd (in both cases $\sum b\left(v_{i}\right)=0$ is satisfied), we find contradiction to Equation (4.6). The contradiction implies that no function $f \in F^{*}(G, A)$ satisfies (4.4) for the constructed $b$.

Case 2: Suppose that $A=Z_{2} \times Z_{2}$. Then Equation (4.5) above has solution $\left\{f_{i 1}^{*}, f_{i 2}^{*}\right\}=\left\{a_{1}, a_{3}\right\}$. Without loss of generality, we may assume that for $1 \leq i \leq k, f_{i 1}^{*}=a_{1}$, and for $k+1 \leq i \leq m, f_{i 1}^{*}=a_{3}$. It follows by (4.4) that

$$
b\left(v_{1}\right)=-k a_{1}-(m-k) a_{3}=k a_{2}+m a_{3},
$$

where we have used the fact that $a_{i}=-a_{i}(i=1,2,3)$ and $a_{1}+a_{3}=a_{2}$. When $m$ is even, Equation (4.7) implies that $b\left(v_{1}\right)=k a_{2}=a_{2}$ or 0 . If we set $b\left(v_{1}\right)=a_{1}=b\left(v_{2}\right)$, we get a contradiction. When $m$ is odd, Equation (4.7) implies that $b\left(v_{1}\right)=k a_{2}+a_{3}=a_{1}$ or $a_{3}$. If we set $b\left(v_{1}\right)=0$ and $b\left(v_{2}\right)=a_{2}$, we also get a contradiction. The contradiction implies that no function $f \in F^{*}(G, A)$ satisfies (4.4) for the constructed $b$.

Case 1 and 2 show that $K_{m, 2}$ is neither $Z_{4}$-connected nor $Z_{2} \times Z_{2^{-}}$ connected. Thus, Equation (4.3) must hold.

Theorem 4.4 now follows by combining Equation (4.2) and Equation (4.3). 


\subsection{The Group Connectivity Number of $K_{m, 3}$}

The main purpose of this section is to determine $\Lambda_{g}\left(K_{m, 3}\right)$.

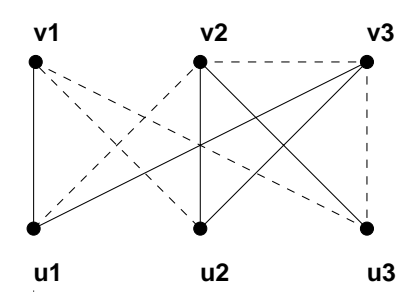

Figure 4.2: $K_{3,3}$

Theorem $4.5 \Lambda_{g}\left(K_{3,3}\right) \leq 4$.

Proof. If we add an edge $\left(v_{2}, v_{3}\right)$ to the graph $K_{3,3}$, (as depicted in Figure 4.2) we can find two edge-disjoint spanning trees $T_{1}$ and $T_{2}$ with $E\left(T_{1}\right)=$ $\left\{v_{1} u_{1}, u_{1} v_{3}, v_{3} u_{2}, u_{2} v_{2}, v_{2} u_{3}\right\}$ and $E\left(T_{2}\right)=\left\{u_{1} v_{2}, v_{2} v_{3}, v_{3} u_{3}, u_{3} v_{1}, v_{1} u_{2}\right\}$. Therefore by Theorem 2.5, $K_{3,3}$ is collapsible, and so by Lemma $2.6, \Lambda_{g}\left(K_{3,3}\right) \leq 4$.

Theorem 4.6 $\Lambda_{g}\left(K_{m, 3}\right)=4$ for any integer $m \geq 3$.

Proof. By Corollary of Theorem 4.3, we obtain an upper bound for $\Lambda_{g}\left(K_{m, 3}\right)$ when $m \geq 3$ as follows

$$
\Lambda_{g}\left(K_{m, 3}\right) \leq \Lambda_{g}\left(K_{3,3}\right) \leq 4
$$

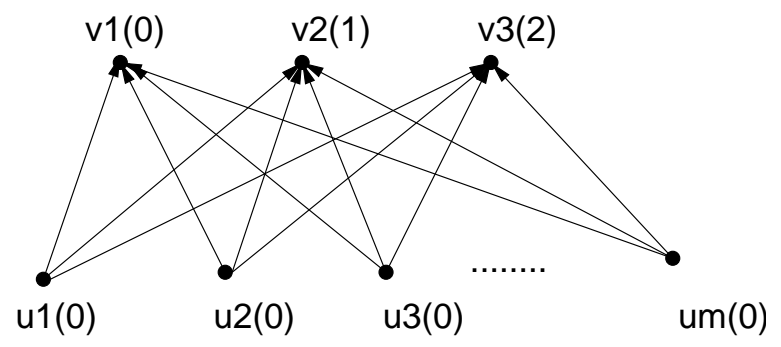

Figure 4.3: $K_{m, 3}$

Just as what was done in the previous section, we next show

$$
\Lambda_{g}\left(K_{m, 3}\right)>3
$$


By Inequality (4.8), it suffices to show that $K_{m, 3} \notin\left\langle Z_{3}\right\rangle$. We shall use the notation in Figure 4.3 and denote $Z_{3}=\{0,1,2\}$. We construct a function $b \in Z\left(K_{m, 3}, Z_{3}\right)$ such that for each $i=1,2, \ldots, m, b\left(u_{i}\right)=0 \in Z_{3}$. For $j=1,2,3$ and for $i=1,2, \ldots, m$, denote $f_{i j}^{*}=f\left(u_{i}, v_{j}\right)$. Then

$$
b\left(u_{i}\right)=f_{i 1}^{*}+f_{i 2}^{*}+f_{i 3}^{*}=0 .
$$

Further notice that in $Z_{3}$, for each $i=1,2, \ldots, m$, the Equation (4.10) above has only two possible solutions:

$$
\text { either } \quad f_{i 1}^{*}=f_{i 2}^{*}=f_{i 3}^{*}=1 \quad \text { or } \quad f_{i 1}^{*}=f_{i 2}^{*}=f_{i 3}^{*}=2 .
$$

In either case, we find that the flows on all three edges connecting these vertices are the same. It follows that the net out flow of the other set of three vertices $v_{1}, v_{2}$ and $v_{3}$ should equal:

$$
\begin{aligned}
& b\left(v_{1}\right)=-\sum_{i=1}^{m} f_{i 1}^{*}=-\sum_{i=1}^{m} f_{i 2}^{*}=b\left(v_{2}\right) \\
& b\left(v_{1}\right)=-\sum_{i=1}^{m} f_{i 1}^{*}=-\sum_{i=1}^{m} f_{i 3}^{*}=b\left(v_{3}\right)
\end{aligned}
$$

Let $b \in Z\left(K_{m, 3}, Z_{3}\right)$ be such that $b\left(u_{i}\right)=0, i=1,2, \ldots, m$ and $b\left(v_{1}\right)=0$, $b\left(v_{2}\right)=1$ and $b\left(v_{3}\right)=2$. Then (4.12) and (4.13) will be violated, leading to a contradiction.

Theorem 4.6 now follows by combining Equation (4.8) and Equation (4.9).

\subsection{The Group Connectivity Number of $K_{m, n}$,$$
(m \geq n \geq 4)
$$

We first determine $\Lambda_{g}\left(K_{4,4}\right)$.

Theorem $4.7 \Lambda_{g}\left(K_{4,4}\right) \leq 3$.

The proof is based on SP contractions we introduced in the previous chapter. The process is depicted in Figure 4.4. Let $A$ be an abelian group with $|A| \geq 3$ and let $G$ denote a graph isomorphic to $K_{4,4}$. First, we apply SP on vertex $v_{1}$; edges $\left(v_{1}, u_{3}\right)$ and $\left(v_{1}, u_{4}\right)$ are changed into edge $\left(u_{3}, u_{4}\right)$. Then apply another SP on vetex $v_{2}$; edges $\left(v_{2}, u_{2}\right)$ and $\left(v_{2}, u_{3}\right)$ are changed into edge $\left(u_{2}, u_{3}\right)$. We end up with the new graph $G^{\prime}$ in (b). $\Lambda_{g}\left(K_{4,4}\right) \leq$ 
$\Lambda_{g}\left(G^{\prime}\right)$ by Theorem 3.1. From (b) to (c), we contract an induced subgraph $H=G\left[\left\{u_{2}, u_{3}, u_{4}, v_{3}, v_{4}\right\}\right]$ into a single vertex $v_{H}$. Notice that subgraph $H$ itself is isomorphic to a wheel $W_{4}$ with its center at $u_{3}$ (see (f)). By Lemma 2.7, $H \in\langle A\rangle$. Further reduction of (c) involves contracting a subgraph induced by $\left\{u_{1}, v_{H}\right\}$ (which forms a cycle $C_{2}$, also known to belong to $\langle A\rangle$ by Lemma 2.4). Reduce (d) by contracting the $C_{2}$ induced by $\left\{v_{1}, u_{1} v_{H}\right\}$. The resulting graph shown in (e) is in $\langle A\rangle$ by Lemma 2.4. Then by Proposition 2.3, $G^{\prime} \in\langle A\rangle$. Thus $\Lambda_{g}\left(K_{4,4}\right) \leq \Lambda_{g}\left(G^{\prime}\right) \leq 3$ by Theorem 3.1.

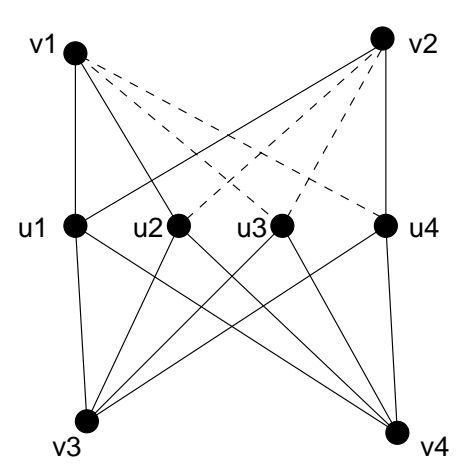

(a) The graph $G$

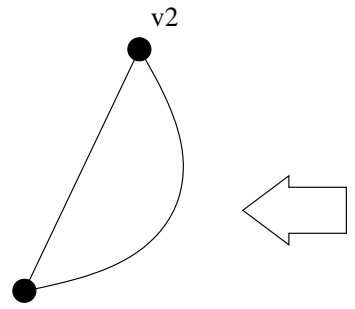

(e)

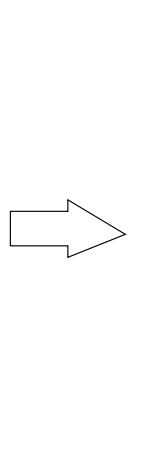

v3

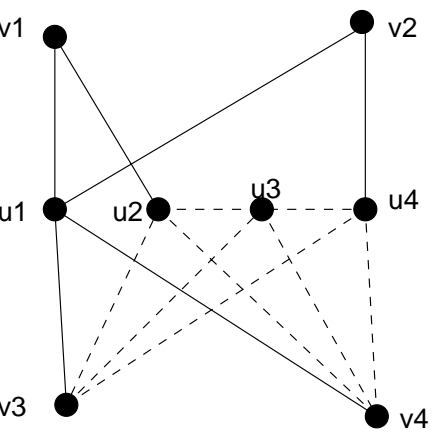

(b) The graph G'

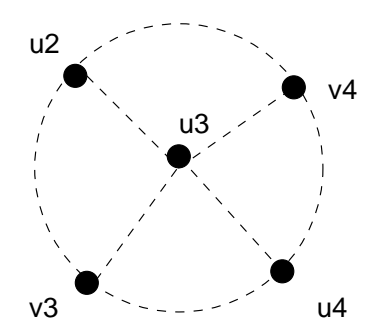

(f) The graph $\mathrm{H}$

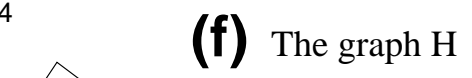


We thus have

Theorem $4.8 \Lambda_{g}\left(K_{m, n}\right)=3$ for any $m \geq n \geq 4$. 


\section{Chapter 5}

\section{Chordal Graphs}

A graph $G$ is chordal if every cycle of length greater than 3 possesses a chord. That is, every induced cycle of $G$ has length at most 3. By Proposition 2.3 and Lemma 2.4, it is easy to see that $\Lambda_{g}(G) \leq 4$.

In this chapter we investigate the connected chordal graphs. We characterize 3-connected chordal graph $G$ with $\Lambda_{g}(G)=3$ and connected chordal graph $G$ with $\Lambda_{g}(G)=4$.

First we introduce some results.

Lemma 5.1 (Lai [11]) Let $A$ be an Abelian group of order at least 3. If $G$ is a 4-edge-connected chordal graph, then $G \in\langle A\rangle$.

Lemma 5.2 (Lai [11]) Let $G$ be a 3-edge-connected chordal graph. Then one of the following holds:

(1.) $G$ is A-connected, for any Abelian group $A$ with $|A| \geq 3$; or

(2.) $G$ has a block isomorphic to a $K_{4}$; or

(3.) $G$ has a subgraph $G_{1}$ such that $G_{1} \notin\left\langle Z_{3}\right\rangle$ and such that $G=G_{1} \oplus K_{4}$.

In Chapter 2, we note $\kappa(G) \leq \kappa^{\prime}(G)$, so we give some results on chordal graphs with $\kappa(G)=3,2$ and 1 .

The main results of this chapter are as follows.

Theorem 5.3 Let $G$ be a 3-connected chordal graph. Then $\Lambda_{g}(G)=3$ if and only if $G \neq K_{4}$.

Proof. $\quad(\Leftarrow)$ Let $A$ be an abelian group with $|A| \geq 4$. We shall first show that every 2 -connected chordal graph $G$ is $A$-connected. Since $G$ is 
chordal, every edge $e$ lies in a cycle $C_{e}$ with $\left|E\left(C_{e}\right)\right| \leq 3$. By Lemma 2.4, $C_{e} \in\langle A\rangle$. Since $G$ is 2-connected, we keep contracting $C_{3}$ and $C_{2}$, finally we get a single vertex which is also $A$-connected. It follows by Proposition $2.3(\mathrm{C} 3)$ that $G \in\langle A\rangle$. Thus $\Lambda_{g}(G) \leq 4$. Therefore, it suffices to show that if $G \neq K_{4}$, and $G$ is a 3 -connected chordal graph, then $G \in\left\langle Z_{3}\right\rangle$. Since $3 \leq \kappa(G) \leq \kappa^{\prime}(G)$. By Lemma 5.1 we only need to show that it is true when $\kappa(G)=\kappa^{\prime}(G)=3$. By Lemma 5.2 we may assume that Lemma 5.2(1) does not hold. If Lemma 5.2(2) holds, then $G$ has a block isomorphic to $K_{4}$ and so $G$ has a cut vertex (see Figure 5.1). Thus $\kappa(G)=1$ in this case, contrary to the assumption that $\kappa(G)=3$.

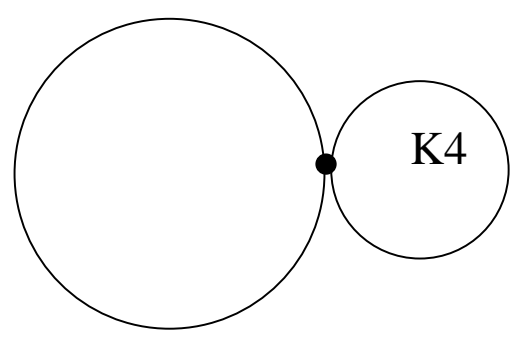

Figure 5.1: Case 2 in Lemma 5.2

If Lemma 5.2(3) holds, then $G$ has a subgraph $G_{1}$ such that $G_{1} \notin\left\langle Z_{3}\right\rangle$ and $G=G_{1} \oplus K_{4}$ (see Figure 5.2). It is obvious that $\kappa(G)=2$, which is a contradiction to the assumption that $\kappa(G)=3$. These contradictions establish the theorem.

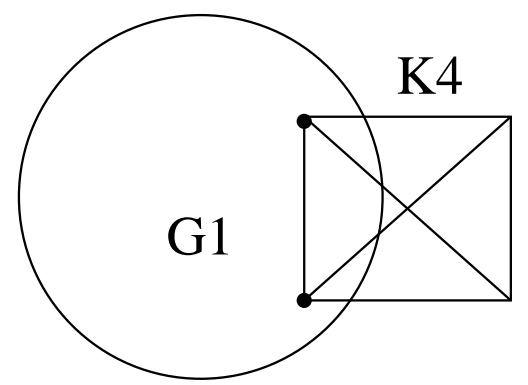

Figure 5.2: Case 3 in Lemma 5.2

$(\Rightarrow)$ Suppose $\Lambda_{g}(G)=3$, then $G \varsubsetneqq K_{4}$ by Lemma 2.10 .

Lemma 5.4 ([5]) Let $G$ be a 2-connected chordal graph and let $\{a, b\}$ be a vertex cut of $G$. Then $a b \in E(G)$. 
Proof. See Figure 5.3. First we prove that there must an edge between the cut vertex $a$ and $b$. Suppose $V^{\prime}$ is a cut vertex set with element $a$ and $b, G_{1}$ and $G_{2}$ being the connected components of $G_{V-V^{\prime}}$ containing $v$ and $u$, respectively. Since $V^{\prime}$ is the minimal vertex separator set, $a \in V^{\prime}$ is adjacent to some vertex in $G_{1}$ and some vertex in $G_{2}$. Therefore, for $a, b \in V^{\prime}$, there exist path $\left[a, v_{1}, \ldots, v_{r}, b\right]$ and $\left[b, u_{1}, \ldots, u_{t}, a\right]$ where each $v_{i} \in G_{1}$ and $u_{i} \in G_{2}$, such that these paths are chosen with smallest possible length. It follows that $\left[a, v_{1}, \ldots, v_{r}, b, u_{1}, \ldots, u_{t}, a\right]$ is a simple cycle whose length is at least 4 , since it is a connected chordal graph, it must have a chord. But $v_{i} u_{j} \notin E(G)$, by the definition of vertex cut, and $v_{i} v_{j} \notin E\left(G_{1}\right)$ and $u_{i} u_{j} \notin E\left(G_{2}\right)$ by the minimality of $r$ and $t$. Thus the only possible chord is $a b \in E(G)$.

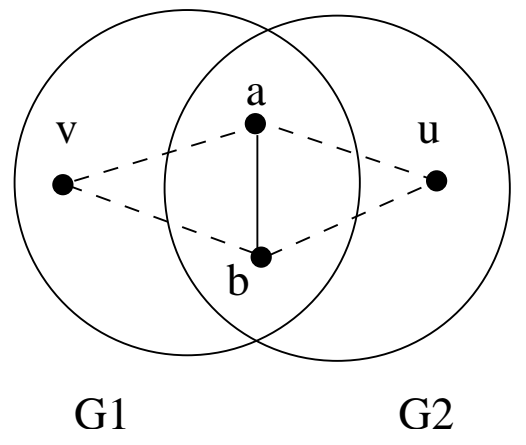

Figure 5.3: 2-connected chordal graph

Theorem 5.5 Let $G$ be a 2-connected chordal graph. Then $\Lambda_{g}(G)=4$ if and only if $G \in\left\{K_{3}, K_{4}\right\}$ or $G$ has two subgraphs $G_{1}$ and $G_{2}$ such that both $\Lambda_{g}\left(G_{1}\right)$ and $\Lambda_{g}\left(G_{2}\right)$ are 4 , and such that $G=G_{1} \oplus G_{2}$.

Proof. By Lemma 2.13, $\Lambda_{g}\left(K_{3}\right)=4=\Lambda_{g}\left(K_{4}\right)$. Suppose first that $G$ has two subgraphs $G_{1}$ and $G_{2}$ such that $\Lambda_{g}\left(G_{1}\right)=\Lambda_{g}\left(G_{2}\right)=4$ and such that $G=G_{1} \oplus G_{2}$. Then by Lemma $3.4, \Lambda_{g}(G)=4$.

Conversely, we assume that $\Lambda_{g}(G)=4$, and that $G \notin\left\{K_{3}, K_{4}\right\}$. If $\kappa(G) \geq 3$, then by Theorem $5.3, \Lambda_{g}(G)=3$. Hence $G$ must have a vertex cut $\{a, b\}$. By Lemma 5.4, we know that $a b \in E(G)$.

Since if $\Lambda_{g}\left(G_{1}\right) \leq \Lambda_{g}\left(G_{2}\right)=3$, then by Proposition 2.3, $\Lambda_{g}(G)=3$. So we only need to show when $\Lambda_{g}\left(G_{1}\right)=4$ and $\Lambda_{g}\left(G_{2}\right)=3, \Lambda_{g}(G)=3$.

Since $G$ is chordal, every edge of $G$ lies in a cycle of length at most 3, and so by Lemma 2.4, for any abelian group $A$ with $|A| \geq 4, G \in\langle A\rangle$. Therefore, it suffices to examine if $G \in\left\langle Z_{3}\right\rangle$. 
Since $\Lambda_{g}\left(G_{2}\right)=3, G_{2} \in\left\langle Z_{3}\right\rangle$. Let $G^{\prime}=G / G_{2}$ and let $e=a b$ denote the edge in both $G_{1}$ and $G_{2}$. Then $G^{\prime}=G / G_{2}=G_{1} / e$. Since $G_{1}$ is a triangulate connected graph, by Lemma $2.14, G_{1} / e$ must be in $\left\langle Z_{3}\right\rangle$. Since $G / G_{2}=G_{1} / e \in\left\langle Z_{3}\right\rangle$ and $G_{2} \in\left\langle Z_{3}\right\rangle$, by Proposition 2.3, $G \in\left\langle Z_{3}\right\rangle$.

Theorem 5.6 Let $G$ be a 2-edge connected chordal graph such that $G$ is not 2-connected. Then $\Lambda_{g}(G)=4$ if and only if for some proper subgraphs $G_{1}$ and $G_{2}$ of $G, \Lambda_{g}\left(G_{1}\right)=4$ or $\Lambda_{g}\left(G_{2}\right)=4$, where $G$ is obtained from $G_{1}$ and $G_{2}$ by identify one fixed vertex in $G_{1}$ with a fixed vertex in $G_{2}$.

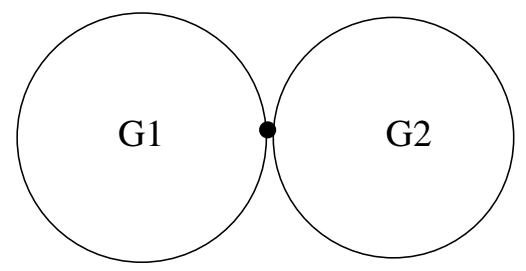

Figure 5.4: 1-connected chordal graph

Proof. See Figure 5.4. Let $A$ be an abelian group with $|A| \geq 4$. By the assumption of Theorem 5.6, $G$ has a cut vertex $v$. Hence $G$ has two connected subgraph $G_{1}$ and $G_{2}$ such that $\left|V\left(G_{i}\right)\right| \geq 2 \quad(i=1,2), V\left(G_{1}\right) \cap V\left(G_{2}\right)=\{v\}$ and $G=G_{1} \cup G_{2}$. Therefore, $G_{1} \cong G / G_{2}$, and $G_{2} \cong G / G_{1}$. If both $G_{1}$ and $G_{2}$ are in $\left\langle Z_{3}\right\rangle$, then by Proposition 2.3, $G \in\left\langle Z_{3}\right\rangle$ also, and so $\Lambda_{g}(G) \leq 3$. It follows that if $\Lambda_{g}(G)=4$, then $\Lambda_{g}\left(G_{1}\right)=4$ or $\Lambda_{g}\left(G_{2}\right)=4$. Conversely suppose $\Lambda_{g}(G)=3$, then by Proposition 2.3(C2), both $G_{1} \cong G / G_{2} \in\left\langle Z_{3}\right\rangle$ and $G_{2} \cong G / G_{1} \in\left\langle Z_{3}\right\rangle$. Hence if $\Lambda_{g}\left(G_{1}\right)=4$ or $\Lambda_{g}\left(G_{2}\right)=4$, then we must also have $\Lambda_{g}(G)=4$. 


\section{Chapter 6}

\section{The Biwheels $B_{n}$}

In this chapter we investigate the problem of group connectivity number for $B_{n}$. The biwheel $B_{n}$ is a graph obtained from a chordless cycle on $n$ edges by adding two additional vertices and joining these two new vertices to all the vertices on the cycle (see Figure 6.1). Shahmohamad [19] gave the following results on minimum flow number of biwheels:

Lemma 6.1 ([18], [19]) Let $n$ be a positive integer.

(1) $\lambda_{g}\left(B_{2 n+1}\right)=2$, for $n \geq 1$.

(2) $\lambda_{g}\left(B_{2 n}\right)=3$, for $n \geq 2$.

We generalized these results to the group connectivity number of biwheels as follows:

Theorem 6.2 The GCN of biwheel $B_{n}$ is $\Lambda_{g}\left(B_{n}\right)=3$.

Proof. Since every edge of $B_{n}$ lies in a $C_{3}$, by Lemma 2.4 and Proposition 2.3 , we can contract $C_{3}$ and $C_{2}$ to show that $B_{n} \in\langle A\rangle$ for any abelian group $A$ with $|A| \geq 4$. Hence it suffices to show that $B_{n} \in\left\langle Z_{3}\right\rangle$. We will prove this by considering two cases.

Case 1: If $n$ is even, by Lemma 2.8 we know that $W_{n} \in\left\langle Z_{3}\right\rangle$. We view that $W_{n}$ as a subgraph of $B_{n}$. By subgraph contraction $B_{n} / W_{n}$ we will have a vertex joint with another (the wheel on $n$ vertices will contract to be one single vertex) by an even number of multiple edges, which is a graph belongs to $Z_{3}$ by Proposition 4.2. Therefore $B_{n} \in\left\langle Z_{3}\right\rangle$ by Proposition 2.3(C3).

Case 2: If $n$ is odd, it is enough to show that $\Lambda_{g}\left(B_{n}\right) \leq 3$.

Let $B_{n}^{\prime}$ be a graph obtained from $B_{n}$ by deleting edges $e_{1}$ and $e_{2}$, and adding edge $e_{3}$ (see Figure 6.1). Actually, $B_{n}^{\prime}$ is obtained by applying SP 
reduction (introduced in Chapter 3 ) to the vertex with label $1, e_{1}$ and $e_{2}$ are replaced by $e_{3}$. By Theorem 3.1, we have $\Lambda_{g}\left(B_{n}\right) \leq \Lambda_{g}\left(B_{n}^{\prime}\right)$.

Now we will show $\Lambda_{g}\left(B_{n}^{\prime}\right) \leq 3$. Observe that $B_{n}^{\prime}$ has a induced subgraph isomorphic to $W_{n-1}$. View this subgraph a $W_{n-1}$. By Lemma $2.8, W_{n-1} \in$ $\left\langle Z_{3}\right\rangle$. We only need to show that $B_{n}^{\prime} / W_{n-1} \in\left\langle Z_{3}\right\rangle$ by Proposition 2.3. Since vertex $a$ and vertex $b$ are joined by an even number of multiple edges in the graph $B_{n}^{\prime} / W_{n-1}$ (see Figure 6.1). Keep contracting $C_{2}$ which is also belongs to $\left\langle Z_{3}\right\rangle$ in $B_{n}^{\prime} / W_{n-1}$ we get a single vertex, which is in $\left\langle Z_{3}\right\rangle$ by Proposition 2.2. It follows by Proposition 2.3 (C3) that $B_{n}^{\prime} / W_{n-1} \in\left\langle Z_{3}\right\rangle$.

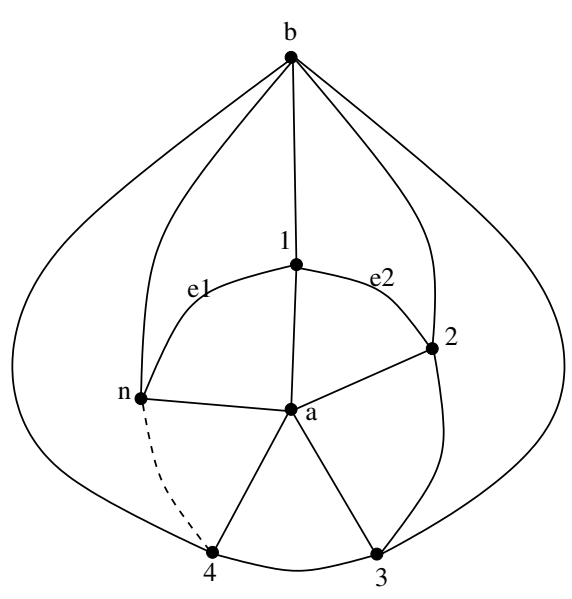

$\mathrm{Bn}$

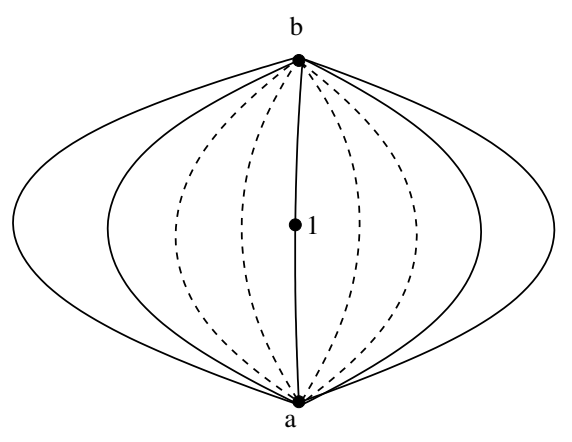

$\mathrm{Bn}$ 'Wn-1

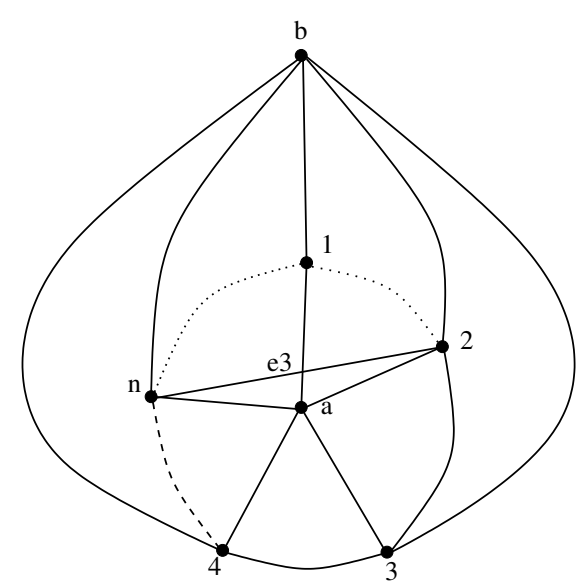

$\mathrm{Bn}$ '

Figure 6.1: Biwheel $B_{n}$ when $n$ is odd 


\section{Chapter 7}

\section{General Graphs}

The main conjectures in this area are the following:

Conjecture 7.1 (Tutte) (3-flow-conjecture) Every 5-edge-connected graph has a nowhere-zero 3-flow.

Conjecture 7.2 (Tutte) (4-flow-conjecture) Every bridgeless graph containing no subdivision of the Peterson graph admits a nowhere-zero 4-flow.

Conjecture 7.3 (Tutte [20]) (5-flow-conjecture) Every bridgeless graph has a nowhere-zero 5-flow. or a weaker conjecture: Every 3-edge-connected graph is in $\left\langle\mathbf{A}_{5}\right\rangle$.

Conjecture 7.4 (Jaeger et al. [8])) Every 5-edge-connected graph $G$ satisfies $\Lambda_{g}(G) \leq 3$.

Conjecture 7.5 (Jaeger et al. [8])) Every 3-edge-connected graph $G$ satisfies $\Lambda_{g}(G) \leq 5$.

Conjecture 7.5 would be best possible if valid, as shown by Lemma 2.4. Conjecture 7.5 is in fact stronger than Conjecture 7.3, as the nowhere zero 5 -flow problem of 2-edge-connected graphs can be routinely reduced to 3edge-connected graphs. However, there exists an infinite family of 4-edgeconnected graph that are not $Z_{3}$-connected (Example 4.3 of [11]), indicating that Conjecture 7.4 will be best possible if it is valid.

By now, the following have been obtained. As stated in Chapter $2, \kappa^{\prime}(G)$ stands for the edge-connectivity of a graph $G$.

Theorem 7.6 (Jeager et al. [8]) Every 3-edge-connected graph $G$ satisfies $\Lambda_{g}(G) \leq 6$. 
The famous theorem by Seymour is as follows:

Theorem 7.7 (Seymour [17]) Let $G$ be a graph with $\kappa^{\prime}(G) \geq 2$, then $G$ admits a nowhere zero 6-flow.

Theorem 7.8 (Jeager et al. [8]) Let $G$ be a graph with $\kappa^{\prime}(G) \geq 4$, then for any $A$ with $|A| \geq 4, G \in\langle A\rangle$.

Theorem 7.9 (Lai [10]) Let $G$ be a collapsible graph and let $A$ be an Abelian group with $|A|=4, G \in\langle A\rangle$.

Theorem 7.10 (Lai and Zhang [12]) Every 3-edge-connected planar graph $G$ satisfies $\Lambda_{g}(G) \leq 5$.

Theorem 7.11 (Lai and Li [13]) Every 5-edge-connected planar graph G satisfies $\Lambda_{g}(G) \leq 3$. 


\section{Chapter 8}

\section{Algorithms}

In this chapter, for the given graph $G=(V, E)$, we denote the number of vertices of $G$ by $n$ and the number of edges of $G$ by $m$.

Algorithm 1: Determine the GCN of a complete bipartite graph $G$.

This algorithm consists of two parts. First, we use Breadth-first search(BFS)

[3] to determine whether $G$ is bipartite. The second part is to determine whether this bipartite is complete, then we can decide the corresponding GCN of the complete bipartite graph based on the results in Chapter 4 .

Part I:

1. Let $s \in V$, set color(s):=gray.

2. For all vertices $u \in V-s$, set $\operatorname{color}(\mathrm{u}):=$ white.

3. Let $\mathrm{Q}$ be a Queue, set the initial value of $\mathrm{Q}$ to be $\{s\}$.

4. Let $\mathrm{D}$ be a array, $\mathrm{D}(\mathrm{u})$ is the shortest distance between $\mathrm{u}$ and $\mathrm{s}$. Set $\mathrm{D}(\mathrm{s}):=0$.

5. While Q is not empty

6. $\quad$ Let $u \in \operatorname{Head}(Q)$.

7. $\quad$ For each $v \in \operatorname{Adj}(u)$

8. $\quad$ IF color $(\mathrm{v})$ is white then

9. $\quad \mathrm{d}(\mathrm{v}):=\mathrm{d}(\mathrm{u})+1 ; \operatorname{color}(\mathrm{v}):=$ grey; Enqueue $(\mathrm{Q}, \mathrm{v})$

10. ENDIF

11. IF ( color $(\mathrm{v})$ is gray ) and $(\mathrm{d}(\mathrm{v})$ is same as $\mathrm{d}(\mathrm{u}))$ then

12. $G$ is not bipartite. Stop

13. ENDIF

14. ENDFOR

15. Dequeue $(\mathrm{Q})$; Color $(\mathrm{u}):=$ black

16. ENDWhile

Part II. 
1. Let A be the vertex set such that $d(v)$ is even, and B be the vertex set such that $\mathrm{d}(\mathrm{v})$ is odd.

2. Let $a:=|A|, b:=|B|$.

3. If for all $v \in A,|\operatorname{adj}(v)|==b$ and $|\operatorname{adj}(u)|==a$ for all $u \in B$ then $\mathrm{G}$ is complete bipartite.

4. If $\operatorname{Min}(a, b)=2$, the GCN of $G$ is 5 according to Theorem 4.4.

5. If $\operatorname{Min}(a, b)=3$, the GCN of $G$ is 4 according to Theorem 4.6.

6. If $\operatorname{Min}(a, b)=4$, the GCN of $G$ is 3 according to Theorem 4.8.

8. end;

Complexity: This algorithm requires $O(n+m)$ time since complexity of algorithm BFS is $O(n+m)$.

Correctness: It is obvious by Theorems in Chapter 4 .

Algorithm 2: Determine the GCN of a chordal graph $G$.

Note that for a chordal graph $G, \Lambda_{g}(G) \leq 4$. First we use Lexicographic breadth-first search [16] to tell whether the graph is chordal or not. Then we find the vertex-connectivity of the chordal graph using the method in [6]. We can decide the corresponding GCN by theorems in Chapter 5 .

1. Apply the Lex BFS based chordal graph recognition algorithm [16] to test $G$ is a chordal graph. If $G$ is not a chordal graph, go to end. Otherwise , go to (2).

2. Use the method in [6] to determine vertex connectivity $\kappa(G)$ of an $n$-vertex and $m$-edge graph $G$.

3. If $\kappa(G) \geq 4$, then $\kappa^{\prime}(G) \geq 4$ and Lemma 5.1 implies that GCN of $G$ is 3 , go to the end.

4. If $\kappa(G)=3$, then GCN of $G$ is 3 , unless $G \cong K_{4}$ (by Theorem 5.3), go to the end.

5. If $\kappa(G)=2$, apply Theorem 5.5. For each edge $e$ of $G$, check if $e$ is a cut-edge and, if so, recursively call Algorithm 2 (beginning at step 2) to determine the GCN of $G_{1}$ and $G_{2}$, go to end.

6. If $\kappa(G)=1$, apply Theorem 5.6. For each vertex $v$ of $G$, recursively call Algorithm 2 (beginning at step 2) to determine the GCN of $G_{1}$ and $G_{2}$, go to end.

end;

Complexity: Step 1 Lex BFS requires $O(n+m)$ time [16]. Step 2 requires $O\left((n-\delta-1+\delta(\delta-1) / 2) m n^{2 / 3}\right)$ time to determine vertex connectivity of an $n$-vertex and $m$-edge graph $G$ [6]. The depth of the recursion in steps 5 and 6 is $O(n)$. Hence the overall complexity is $O\left(n^{7}\right)$.

Correctness: It is obvious by Theorems in Chapter 5 . 
Algorithm 3: Determine the GCN of a graph with $\kappa^{\prime}(G) \geq 3$.

Our algorithm is based on the classical Theorem 7.6 and Theorem 7.8.

The algorithm is as follows:

Step 1 to step 3 is the algorithm [15] based on the Min-cut problem to determine the edge-connectivity of $G$. And when $|V| \geq 2$, also a mincut $(A, \bar{A})$ of $G$.

1. For $v$ a vertex of minimum degree in $G$ set $S:=v, T:=\{w \mid w$ adjacent to $v\}$, $U:=V-S-T, k=\operatorname{deg}(v), A=v, \bar{A}=V-v$. (We initialize $k=\delta$ with, for $|V| \geq 2$, a corresponding cut that will be a mincut unless $\kappa^{\prime}(G)=|(A, \bar{A})| \leq$ $\delta-1$.)

2. If $U=\emptyset$, set $\kappa^{\prime}(G)=k$ and stop.

3. Choose $v \in U$ and determine a out $(B, \bar{B})$ of minimum cardinality with $S \subset U, v \in \bar{B}$. Set $S:=S \cup v, T:=T \cup\{w \mid w$ adjacent to $v\}$, $U:=U-T-v$. If $|(B, \bar{B})|<k$, then set $k=|(B, \bar{B})|$ and $(A, \bar{A}):=(B, \bar{B})$. Return to step 2.

4. If $\kappa^{\prime}(G)<3$, then go to end.

5. If $\kappa^{\prime}(G)=3$, then the group connectivity number $\Lambda_{g}(G) \leq 6$, go to end.

6. If $\kappa^{\prime}(G) \geq 4$, then the group connectivity number $\Lambda_{g}(G) \leq 4$, go to end.

end;

Complexity: This algorithm require $O(n m)$ time since step 1 to step 3 require $O(n m)$ time to determine edge connectivity of an $n$-vertex and $m$ edge graph $G$ [15].

Correctness: It is obvious by Theorem 7.6 and Theorem 7.8. 


\section{Chapter 9}

\section{Conclusion}

In this paper we have constructed infinite families of graphs with group connectivity number 3,4 and 5 . One open problem is finding a more efficient algorithm to determine the group connectivity number of a chordal graph when $\kappa(G)=1$ or 2 . This reduces to determine whether a chordal graph $G$ with $\kappa(G)=1$ or 2 is $Z_{3}$-connected.

There are many results in general graphs as shown in Chapter 7 . We need more new methods to discover the group connectivity characteristics of more general graphs. 


\section{Bibliography}

[1] J. A. Bondy, U. S. R. Murty, Graph Theory with Applications, American Elsevier, New York, 1976.

[2] P. A. Catlin, A reduction method to find spanning eulerian subgraphs, J. Graph Theory, 12 (1988), 29-44.

[3] T. H. Cormen, C. E. Leiserson and R. L. Rivest, Introduction to Algorithm. The MIT Press Cambridge, Massachusetts/London, England, 1989, 469-476.

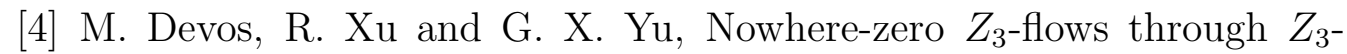
connectivity, submitted to Discrete Math.

[5] G. Dirac, On rigid circuit graphs, Abh. Math. Sem. Univ. Hamburg 25 (1961), 71-76.

[6] A. H. Esfahanian and S. L. Hakimi, On computing the connectivities of graphs and digraphs. Networks (1984), 355-366.

[7] F. Jaeger, Nowhere zero flow problems, in "Selected Topics in Graph Theory", (L. Beineke and R. Wilson, Eds.), vol. 3, pp. 91-95, Academic Press, London/New York, 1988.

[8] F. Jaeger, N. Linial, C. Payan and N. Tarsi, Group connectivity of graphs - a nonhomogeneous analogue of nowhere zero flow properties, J. Combinatorial Theory, Ser. B 56 (1992), 165-182.

[9] H.-J. Lai, Reduction towards collapsibility, Graph theory, combinatorics, and applications, (Y. Alavi and A. Schwenk, Eds.), pp. 661-670, John Wiley and Sons, 1995.

[10] H.-J. Lai, Extending a partial nowhere-zero 4-flow, J. Graph Theory, 30 (1999), 277-288. 
[11] H.-J. Lai, Group connectivity of 3-edge-connected chordal graphs, Graphs and Combinatorics, 16 (2000), 165-176.

[12] H.-J. Lai, X. K. Zhang, Group chromatic number of graphs without $K_{5}$-minors, Graphs and Combinatorics, 18 (2002), 147-154.

[13] H.-J. Lai, X. Li, Group chromatic number of planar graphs with girth at least 4 , submitted.

[14] H.-J. Lai, R. Xu and C. Q. Zhang, Group connectivity of triangularly connected graphs, submitted.

[15] D. W. Matula, Determining edge connectivity in $O(m n)$ time, 28th Symp. on Foundations of Computer Science, (1987), 249-251.

[16] D. J. Rose, R. E. Tarjan and G. S. Lueker, Algorithmic aspects of vertex elimination on graph, SIAM J. Comput., 5 (1976), 266-283.

[17] P. D. Seymour, Nowhere zero 6-flows, J. Combinatorial Theory, Ser. B., 30 (1981), 82-94.

[18] H. Shahmohamad, "On nowhere-zero flows, chromatic equivalence and chromatic equivalence of graphs", PhD Thesis, University of Pittsburg, 2000 .

[19] H. Shahmohamad, On minimum flow number of graphs, Bulletin of the ICA, 35 (2002), 26-36.

[20] W. T. Tutte, A contribution to the theory of chromatic polynomials, Canad. J. Math., 6 (1954), 80-91.

[21] W. T. Tutte, On the imbedding of linear graph into surfaces. Proc. Lond. Math. Soc., II Ser. 51, (1949), 464-483.

[22] C. Q. Zhang, Integer Flows and Cycle Covers of Graphs, Marcel Dekker, Inc., 1997. 